nitive and functional status during the last six months of life. Note that all patients with neuropathological lesions other than those of $\mathrm{AD}$ were excluded from further studies. Using this sample base, we are identifying molecular changes that occur very early in cognitive decline, before a diagnosis of definite $\mathrm{AD}$. We are studying molecular changes in five different brain areas, including the middle frontal gyrus, superior temporal gyrus, the entorhinal cortex, inferior parietal lobule and the primary visual cortex. As part of these studies, we have begun to make use of cDNA arrays for RNA profiling in brain tissue to identify changes in gene expression that occur with the earliest changes in cognition. Our initial focus for RNA profiling is the middle frontal gyrus, an area free of neuropathology in early dementia but where we have identified changes in total amyloid- $\beta$ protein $(A \beta)$ levels. The $A \beta$ we measure represents soluble and perhaps preaggregated $A \beta$ and apparently increases before any neurofibrillary changes or amyloid plaque formation. Our first studies are being carried out in ten controls (CDR 0 ), ten individuals with questionable dementia (CDR 0.5) and ten individuals with mild dementia (CDR 1.0). Note that the controls are age and gender matched, are derived from the same population as the cases and have undergone the same antemortem and post-mortem assessments.

Schizophrenic cases have been selected from our available cohort of 143 antemortem assessed aged schizophrenic subjects. Subjects were derived from the Pilgrim Psychiatric Center, a facility with which the Department collaborates extensively and provides clinical services and antemortem psychiatric assessments. A small subset of cases (10) were identified for preliminary RNA profiling. Among the selection criteria were the absence of neuropathology, short postmortem intervals and a minimum of six neuroleptic free weeks before death. In addition, age, gender and PMI matching with those in the control group for the $\mathrm{AD}$ was used to reduce the total cost of the project. Our studies have begun in the middle frontal gyrus, an area implicated in schizophrenia and being analysed in the AD sample. Later studies will expand this survey to the medial and lateral aspects of the mediodorsal nucleus of the thalamus and the medial and lateral aspects of the pulvinar. These latter areas are the subject of intense study by the Department because of alterations in size and cell number associated with schizophrenia.

For all four groups (control, questionably demented, mildly demented, schizophrenic) RNA profiling has begun making use of cDNA arrays including known genes and sequence-verified ESTs. The cDNA arrays encompass over 7,000 cDNAs, of which over one-half are ESTs. By this combined approach using known genes and sequence-verified ESTs, we will maximize our chances of identifying mRNAs that have consistently altered expression in AD and in schizophrenia. Note that in previous studies in the Department we have made use of differential display for RNA profiling and have identified several mRNAs that are consistently altered in schizophrenia in a manner independent of neuroleptic treatments. One such mRNA, termed SZ-1, represents a very rare transcript that is consistently and profoundly altered in schizophrenia. These results provide important support for the use of cDNA arrays in RNA profiling in neuropsychiatric disorders.

Campbell, Michael

\section{Methods for comparing transcriptional pattems to determine potential clues to biological function}

\author{
Michael Campbell
}

M olecular Applications Group, Palo Alto, California, USA

Human mitosis is both activated and accompanied by genome-wide transcriptional changes, suggesting regulation of diverse biological pathways. Cancer-associated genetic alterations that disrupt the cell cycle often affect these broad transcriptional patterns, identifying discrete biological processes potentially involved in tumour-associated cellular phenotypes. To systematically identify biological processes that may be activated during the human cell cycle, we characterized more than 42,000 transcript levels during mitosis in fibroblasts. To study how tumorigenic conditions might affect a subset of these functions, we also performed this analysis on fibroblasts deficient in retinoblastoma gene product function. To analyse this data, we filtered the data based on the confidence level, clustered the remaining genes and have developed the first algorithms for statistically linking genome-wide transcriptional patterns and biological function. These methods clearly demonstrate association of function with specific transcriptional patterns, and provide means to automatically compare transcriptional patterns to determine potential clues to biological function.

Cary, Robert

\section{Microarray analysis of the DNA-depen- dent protein kinase catalytic subunit knock-out mouse: global effects on gene regulation and the cellular response to ionizing radiation}

\author{
Robert B. Cary ${ }^{1}$, Akihiro Kurimasa' ${ }^{2}$, Konan Peck ${ }^{3} \&$ David J. Chen ${ }^{2}$ \\ ${ }^{1}$ Los Alamos National Laboratory, Life Sciences Division, DNA Damage and \\ Repair Group, Los Alamos, New M exico 87545, USA \\ ${ }^{2}$ Law rence Berkeley National Laboratory, Life Sciences Division, Department of; \\ Radiation Biology and DNA Repair, Berkeley, California 94720, USA \\ ${ }^{3}$ Institute of Biomedical Sciences, Academia Sinica, Taipei 115, Taiwan, ROC
}

We have generated transgenic mice carrying targeted deletions of the DNAdependent protein kinase catalytic subunit (DNA-PKcs) gene. These animals offer the unique opportunity to gain insights into the molecular mechanisms of this kinase in DNA repair and other cellular processes. As a step toward understanding the global effects of DNA-PKcs knock-outs, we have examined the transcriptional consequences of the targeted deletion using a microarray based approach. These data have been generated using cells both exposed and unexposed to low doses of ionizing radiation. By using a combination of wild-type and heterozygous controls we have examined the cellular response to ionizing radiation in homozygous knock-out mice using a 9600 element human cDNA microarray coupled with a colorimetric detection scheme. Cross-species hybridisation offers the potential to focus on the identification of differentially regulated transcripts of high conservation between these two recently diverged species. Our results are presented and discussed in light of the known genetics and biology of the DNAPK pathway of DNA repair.

Causton, Helen

\section{Functional redundancy of two transcrip- tional coactivator complexes}

\author{
Helen C. Causton ${ }^{1}$, Tong Ihn Lee ${ }^{1,2}$, Frank C.P. Holstege ${ }^{1}$, Ezra \\ Jennings ${ }^{1,2} \&$ Richard A. Young ${ }^{1,2}$ \\ ${ }^{1}$ W hitehead Institute for Biomedical Research, Nine Cambridge Center, \\ Cambridge, M assachusett, 02142, USA \\ ${ }^{2}$ Department of Biology, M assachusetts Institute of Technology, Cambridge, \\ M assachusetts 02139, USA
}

Genome-wide expression analysis is being used to study the extent to which various components of the transcription machinery are involved in gene expression in vivo and to identify the contributions of specific subunits of these complexes to gene regulation. Among the multi-subunit coactivator complexes described in eukaryotes, TFIID and SAGA are among the best studied and most conserved, yet 